\title{
Mortality and acute exacerbation of COPD: a pilot study on the influence of myocardial injury
}

To the Editor:

Acute exacerbations of chronic obstructive pulmonary disease (COPD) punctuate important disease progression [1]. In-hospital mortality rates for acute exacerbations of COPD vary between $2.5 \%$ to $24.5 \%$ [2-4]. An integrated clinical score, CURB-65, has been proposed to predict in-hospital and 30-day mortality in acute exacerbations of COPD $[5,6]$. According to death-certificate data the most common proximate cause of death in COPD is cardiac disease [7]. An association between elevated cardiac high-sensitivity troponin (hs-cTn) at admission and mortality has been reported in acute exacerbations of COPD $[8,9]$. Copeptin has also been associated with poor clinical outcomes and mortality of acute exacerbations of COPD [10], as well as pneumonia [11] and myocardial infarction [12]. Recently, the combination of elevated copeptin and hs-cTn at admission for acute chest pain has been shown to have better discriminative value for acute myocardial injury [13] than troponin alone, as well as strong prognostic value for major cardiac adverse events [14]. We hypothesised that myocardial injury may be an important cause of death in patients admitted with an acute exacerbations of COPD. Accordingly, we investigated whether circulating markers of myocardial injury at the time of presentation to the emergency department (ED) with acute exacerbations of COPD added prognostic value to the CURB-65 score.

Initial results were derived in a discovery cohort of 356 consecutive adult patients with acute exacerbations of COPD who presented to the EDs at the Christchurch Heart Institute (New Zealand, $\mathrm{n}=160$ ), Lariboisière Hospital in Paris (France, $\mathrm{n}=112$ ), Monastir University Hospital (Tunisia, $\mathrm{n}=55$ ) and Sivas University Hospital (Turkey, $\mathrm{n}=29$ ). Median (interquartile range) age was $72(63-79)$ years and 57\% were male. A prior history of COPD was present in $85 \%$, heart failure in $20 \%$ and hypertension in $41 \%$ of subjects. At admission, the median systolic blood pressure was $140(123-160) \mathrm{mmHg}$, median heart rate was $98(86-112) \mathrm{min}^{-1}$ and median respiratory rate was $26(22-32) \mathrm{min}^{-1}$. The median length of hospital stay was 4 (1-8) days. 44 patients (12.4\%) were admitted to an intensive care unit. 27 patients (7.6\%) died during the 30-day follow-up period. An independent validation cohort comprised consecutive patients with acute exacerbations of COPD prospectively enrolled at the Basel University Hospital (Switzerland, $\mathrm{n}=135$ ). The validation cohort had similar characteristics to the derivation cohort and 30-day mortality was $7.8 \%$.

This study was performed in accordance with the declaration of Helsinki. All patients provided written informed consent.

CURB-65 was calculated by using information collected upon admission to the ED [5]. The CURB-65 score was indicative of low risk (0-1) in $51 \%$ of the derivation cohort, intermediate risk (2) in $32 \%$ and high risk $(\geqslant 3)$ of 30 -day mortality in the remaining $17 \%$. The primary end-point was 30 -day all-cause mortality. Follow-up by phone interview for events to 30 days was completed. Receiver operating characteristic (ROC) curve analysis yielded an area under the curve (AUC) of 0.67 (95\% CI 0.57-0.76) for the prediction of 30-day mortality by using the CURB-65 score.

Blood samples were collected within $4 \mathrm{~h}$ of admission into tubes containing ethylenediaminetetra-acetic acid (EDTA) with immediate separation of plasma, which was stored at $-80^{\circ} \mathrm{C}$ prior to assay. We measured five biomarkers that reflect different pathophysiological pathways: inflammation and/or infection by C-reactive protein (CRP, Abbott laboratories, Abbott Park, IL, USA) and procalcitonin (PCT, Thermo-Fisher B.R.A.H.M.S. AG, Hennigsdorf, Germany); myocardial damage by high-sensitivity cardiac

@ERSpublications

Acute exacerbated COPD patients with elevated markers of myocardial injury are at high risk of early mortality http://ow.ly/aAaM30adU28

Cite this article as: Laribi S, Pemberton CJ, Kirwan L, et al. Mortality and acute exacerbation of COPD: a pilot study on the influence of myocardial injury. Eur Respir J 2017; 49: 1700096 [https://doi.org/10.1183/ 13993003.00096-2017]. 
troponin I (hs-cTnI, Abbott laboratories, Abbott Park, IL, USA), or high-sensitivity cardiac troponin T (hs-cTnT, Roche, Basel, Switzerland) and copeptin (Thermo-Fisher B.R.A.H.M.S. AG, Hennigsdorf, Germany) and cardiac load by B-type natriuretic peptide (BNP, Abbott laboratories, Abbott Park, IL, USA).

Mean plasma concentrations of cardiovascular biomarkers were elevated in patients with acute exacerbations of COPD at admission. Elevated plasma hs-cTnI was found in $36.5 \%$ patients, elevated copeptin in $27.5 \%$ and the combination of elevated hs-cTnI and copeptin in $13.8 \%$. BNP was elevated in $41 \%$, PCT in $9 \%$ and CRP in $70.2 \%$ of patients.

Hs-cTnI, copeptin, BNP and PCT (but not CRP) were significantly greater at admission in those who died within 30 days than in survivors.

All biomarkers displayed similar AUC values, sensitivity, specificity, negative predictive value (NPV) and positive predictive value (PPV) for prediction of 30-day mortality as the CURB-65 score.

Multiple logistic regression indicated that three biomarkers: hs-cTnI (HR 1.004, 95\% CI 1.001-0.006; $\mathrm{p}=0.007$ ), copeptin (HR 1.005, 95\% CI 1.001-1.009; $\mathrm{p}=0.023$ ) and BNP (HR 1.001, 95\% CI 1.000-1.001; $\mathrm{p}=0.004$ ) were independently associated with 30-day mortality, while CRP and PCT were not.

Circulating hs-cTnI, copeptin and BNP offered incremental prognostic value beyond CURB-65 in patients with acute exacerbations of COPD. Hs-cTnI improved the reclassification with a total net reclassification improvement (NRI) of 0.378 (95\% CI -0.005 to 0.761 ); total NRI for copeptin was 0.616 (95\% CI 0.213 1.02) and BNP 0.572 (95\% CI 0.167-0.976). Integrated discrimination improvement (IDI) was similar for the three cardiovascular biomarkers: 0.049 for hs-cTnI, 0.053 for copeptin and 0.055 for BNP. More importantly, the combination of hs-cTnI and copeptin had a better total NRI (0.653, 95\% CI 0.241-1.064) and IDI (0.075) than circulating troponin or copeptin alone or the combination of BNP and hs-cTnI (NRI 0.498, 95\% CI 0.103-0.459; and IDI 0.064). Hence, combined elevation of hs-cTnI and copeptin ( $\mathrm{n}=49)$ conferred an especially high risk of being associated with a greater than $20 \%$ mortality, that is threefold greater than mortality in patients with elevated hs-cTnI but normal copeptin plasma concentrations (5.7\%) (figure 1).

These results were confirmed in the Basel cohort where the combination of elevated hs-cTnI (or hs-cTnT) and elevated copeptin was also associated with a much greater 30-day mortality than in patients with isolated elevation of hs-cTnI (or hs-cTnT) but normal copeptin.

Our study showed the striking prognostic value of myocardial injury markers when added to the pulmonary severity score, CURB-65, for prediction of 30-day mortality in acute exacerbations of COPD. Patients included in this analysis were of similar age and BMI, with a similar burden of pulmonary or cardiovascular comorbidities and similar short-term mortality as reported in previously published acute exacerbations of COPD cohorts [3,8]. Our study confirmed the expected association between CURB-65 scores and short-term mortality as previously described $[5,6]$. Our findings are consistent with the reported frequent coexistence of lung and cardiovascular disease in COPD patients [15]. In a meta-analysis of eight studies, elevation of cardiac troponin emerged as an independent predictor of all-cause mortality in acute exacerbations of COPD patients [8]. The current report shows that hs-troponin improves the prognostic value of the pulmonary severity score CURB-65 in prediction of short-term mortality in acute exacerbations of COPD. Our study further shows that elevation of both hs-troponin and copeptin was associated with the worst outcomes. Though mild elevations in hs-troponin may be caused by multiple chronic conditions, copeptin has been suggested to allow separation between those stable chronic

FIGURE 130 -day mortality rate in acute exacerbations of COPD patients as a function of circulating markers of myocardial injury at admission. Of note, there were no deaths in the validation cohort (with either hs-cTnl or hs-cTnTl with elevated high-sensitivity cardiac troponin and normal copeptin.

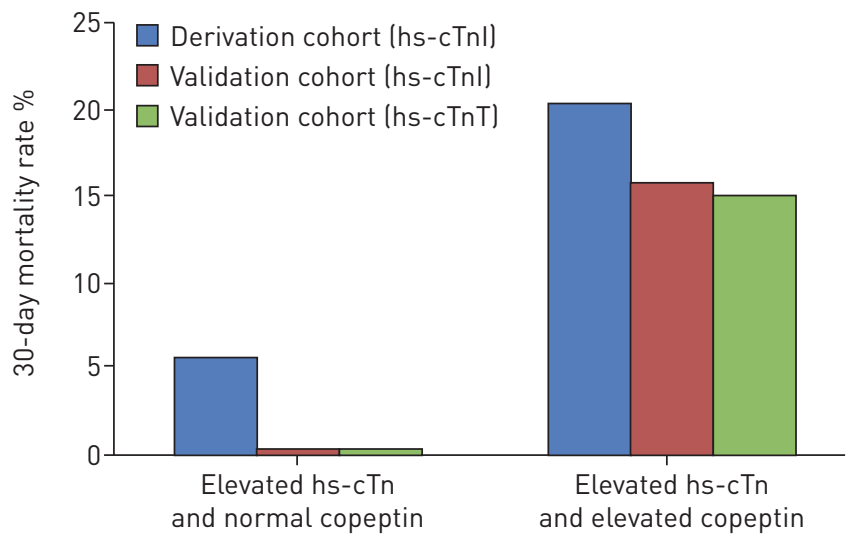


conditions from acute myocardial injury [13]. Accordingly, our study strongly indicates that concurrent elevation of both hs-troponin and copeptin identifies patients with acute exacerbations of COPD with a poor prognosis that potentially reflects important myocardial damage. Limitations to the current study include the absence of data on lung function (e.g. GOLD [1] scores and/or measurements of FEV1) as well as absence of cardiac function (echocardiography at admission).

In summary, our study demonstrated a frequent and striking role of probable acute myocardial injury influencing short-term outcome in acute exacerbations of COPD. Indeed, patients with acute exacerbations of COPD who have elevated markers of myocardial damage are at high risk of early mortality, independent of the severity of lung disease. Future trials should assess whether an intervention strategy algorithm guided by hs-troponin and copeptin levels might improve outcomes.

Said Laribi ${ }^{1,2,3}$, Chris J. Pemberton ${ }^{4}$, Lyndsey Kirwan $^{3}$, Semir Nouira ${ }^{5}$, Kenan Turkdogan ${ }^{6}$, Mehmet Birhan Yilmaz ${ }^{7}$, Richard W. Troughton ${ }^{4}$, Etienne Gayat $^{3,8,9}$, Mercedes Rivas-Lasarte ${ }^{3,10}$, Malha Sadoune, Zaid Sabti ${ }^{11}$, Erwin Hansconrad ${ }^{12}$, Justina Motiejunaite $\oplus^{3,9,13}$, Patrick Plaisance ${ }^{3,8,12}$, Agim Beshiri ${ }^{14}$, Wenjia Chen ${ }^{15}$, Corinne Collet ${ }^{16}$, J. Mark FitzGerald ${ }^{17}$, Christian Mueller ${ }^{11}$, Jean-Marie Launay ${ }^{3,16}$, Mark Richards ${ }^{4,18}$ and Alexandre Mebazaa ${ }^{3,8,9,19}$ on behalf of the GREAT (Global Research on Acute Conditions Team) Network

${ }^{1}$ François-Rabelais University, School of Medicine, Tours, France. ${ }^{2}$ Tours University Hospital, Emergency Medicine Dept, Tours, France. ${ }^{3}$ INSERM, U942, BIOmarkers in CArdioNeuroVAScular diseases, Paris, France. ${ }^{4}$ Christchurch Heart Institute, Dept of Medicine, University of Otago, Christchurch, New Zealand. ${ }^{5}$ Emergency Dept and Research Unit UR06SP21, Fattouma Bourguiba University Hospital, Monastir, Tunisia. ${ }^{6}$ Adnan Menderes University, Faculty of Medicine, Dept of Emergeny Medicine, Aydin, Turkey. ${ }^{7}$ Cumhuriyet University, Faculty of Medicine, Dept of Cardiology, Sivas, Turkey. ${ }^{8}$ Univ Paris Diderot, Sorbonne Paris Cité, Paris, France. ${ }^{9}$ APHP, Saint Louis Lariboisière Hospitals, Dept of Anesthesiology and Critical Care, Paris, France. ${ }^{10}$ Cardiology Dept, Hospital de la Santa CreuiSant Pau, Biomedical Research Institute IIB-SantPau, Universidad Autónoma de Barcelona, Barcelona, Spain. ${ }^{11}$ Dept of Internal Medicine, University Hospital, Basel, Switzerland. ${ }^{12}$ APHP, Saint Louis Lariboisière Hospitals, Emergency Medicine Dept, Paris, France. ${ }^{13}$ Dept of Cardiology, Lithuanian University of Health Sciences Kauno Klinikos, Kaunas, Lithuania. ${ }^{14}$ Abbott Laboratories, Abbott Park, IL, USA. ${ }^{15}$ Institute for Heart and Lung Health, Respiratory Division, University of British Columbia, Vancouver, BC, Canada. ${ }^{16}$ APHP, Saint Louis Lariboisière Hospitals, Dept of Biochemistry, Paris, France. ${ }^{17} \mathrm{UBC}$ and VGH Divisions of Respiratory Medicine, Institute for Heart and Lung Health, Vancouver, BC, Canada. ${ }^{18}$ Cardiovascular Research Institute, National University of Singapore, Singapore. ${ }^{19}$ Vilnius University, Vilnius, Lithuania.

Correspondence: Alexandre Mebazaa, University Paris Diderot, PRES Sorbonne Paris Cité, Department of Anesthesiology and Critical Care Medicine, Hôpitaux Universitaires Saint-Louis-Lariboisière, Assistance PubliqueHôpitaux de Paris (AP-HP), Paris, France. E-mail: alexandre.mebazaa@aphp.fr

Received: Dec 132016 | Accepted after revision: March 202017

Support statement: This study was funded by ThermoFisher. A. Mebazaa and J. Motiejunaite would like to acknowledge the support of the Research Council of Lithuania, grant number MIP-049/2015. Funding information for this article has been deposited with the Crossref Funder Registry.

Conflict of interest: Disclosures can be found alongside this article at erj.ersjournals.com

\section{References}

1 Vestbo J, Hurd SS, Agusti AG, et al. Global strategy for the diagnosis, management, and prevention of COPD: GOLD executive summary. Am J Respir Crit Care Med 2013; 187: 347-365.

2 Patil SP, Krishnan JA, Lechtzin N, et al. In-hospital mortality following acute exacerbations of COPD. Arch Intern Med 2003; 163: 1180-1186.

3 Hartl S, Lopez-Campos JL, Pozo-Rodriguez F, et al. Risk of death and readmission of hospital-admitted COPD exacerbations: European COPD Audit. Eur Respir J 2016; 47: 113-121.

4 Steer J, Norman EM, Afolabi OA, et al. Dyspnoea severity and pneumonia as predictors of in-hospital mortality and early readmission in acute exacerbations of COPD. Thorax 2012; 67: 117-121.

5 Chang CL, Sullivan GD, Karalus NC, et al. Predicting early mortality in acute exacerbation of COPD using the CURB65 score. Respirology 2011; 16: 146-151.

6 Roche N, Zureik M, Soussan D, et al. Predictors of outcomes in COPD exacerbation cases presenting to the emergency department. Eur Respir J 2008; 32: 953-961.

7 Hansell AL, Walk JA, Soriano JB. What do COPD patients die from? A multiple cause coding analysis. Eur Respir J 2003; 22: 809-814.

8 Pavasini R, d'Ascenzo F, Campo G, et al. Cardiac troponin elevation predicts all-cause mortality in patients with acute exacerbation of COPD: Systematic review and meta-analysis. Int J Cardiol 2015; 191: 187-193.

9 Chang CL, Robinson SC, Mills GD, et al. Biochemical markers of cardiac dysfunction predict mortality in acute exacerbations of COPD. Thorax 2011; 66: 764-768.

10 Stolz D, Christ-Crain M, Morgenthaler NG, et al. Copeptin, C-reactive protein, and procalcitonin as prognostic biomarkers in acute exacerbation of COPD. Chest 2007; 131: 1058-1067.

11 Viasus D, Del Rio-Pertuz G, Simonetti AF, et al. Biomarkers for predicting short-term mortality in community-acquired pneumonia: A systematic review and meta-analysis. J Infect 2016; 72: 273-282.

12 Boeddinghaus J, Reichlin T, Nestelberger T, et al. Early diagnosis of acute myocardial infarction in patients with mild elevations of cardiac troponin. Clin Res Cardiol 2017; in press [https://doi.org/10.1007/s00392-016-1075-9]. 
13 Ray P, Charpentier S, Chenevier-Gobeaux C, et al. Combined copeptin and troponin to rule out myocardial infarction in patients with chest pain and a history of coronary artery disease. Am J Emerg Med 2012; 30: 440-448.

14 Möckel M, Searle J, Hamm C, et al. Early discharge using single cardiac troponin and copeptin testing in patients with suspected acute coronary syndrome (ACS): a randomized, controlled clinical process study. Eur Heart J 2015; 36: $369-376$.

15 Chen W, Thomas J, Sadatsafavi M, et al. Risk of cardiovascular comorbidity in patients with COPD: a systematic review and meta-analysis. Lancet Respir Med 2015; 3: 631-639. 